\title{
Synaptic Remodeling Revealed by Repeated In Vivo Observations and Electron Microscopy of Identified Frog Neuromuscular Junctions
}

\author{
Chien-Ping Ko and Lanlin Chen \\ Department of Biological Sciences, Section of Neurobiology, University of Southern California, Los Angeles, California \\ 90089-2520
}

This work aimed to examine the mechanism of synaptic remodeling using repeated in vivo observations, followed by electron microscopy, of identified frog neuromuscular junctions (NMJs). Our previous light microscopic studies suggested that extension of synaptic extracellular matrix (ECM) precedes, and may play a role in, nerve terminal (NT) growth during synaptic remodeling. To test this hypothesis, sartorius muscles were double labeled with a fluorescent dye, 4-(4-diethylamino-styryl)- $N$ methylpyridinium iodide, for NTs and rhodamine-conjugated peanut agglutinin for synaptic ECM. The double-labeled NMJs were observed in vivo with video-enhanced fluorescence microscopy. Two to three months after nerve sprouting was induced by a nerve graft, the same NMJs were restained and reexamined. After the final in vivo observations, the same NMJs were examined with semiserial thin section electron microscopy. Light microscopic observation of NMJs that showed synaptic ECM longer than the NT was confirmed with electron microscopy. At junctional branches where synaptic ECM extended beyond the NT, a Schwann cell process longer than the NT was observed in one example, whereas a Schwann cell with the same length as the NT was seen in other examples. In both cases, junctional folds were absent at the extended ECM region. In contrast, junctional folds were observed at the region vacated by a retracted NT. These results suggest that extension of synaptic ECM and Schwann cell processes may lead, and play a role in, the NT growth during the remodeling of adult synaptic connections.

Key words: nerve sprouting; neuromuscular junctions; extracellular matrix; Schwann cells; electron microscopy; repeated in vivo observations; synaptic remodeling; peanut agglutinin; axonal outgrowth; 4-di-2-Asp
The remodeling of synaptic connections is thought to be ongoing throughout an animal's life (Purves and Lichtman, 1985; Wernig and Herrera, 1986) and may be a basis for memory storage (Bailey and Kandel, 1993). To demonstrate this dynamic feature of adult synaptic connections directly, video-enhanced microscopy can be used to visualize repeatedly the same synapses over a period of time in living animals (for review, see Purves, 1989). Issing this approach, several groups have shown primarily proportionate growth with little, or relatively minor, changes in individual nerve terminal (NT) branches at adult mammalian neuromuscular junctions (NMJs) (Lichtman et al., 1987; Robbins and Polak, 1988; Wigston, 1989, 1990; Balice-Gordon and Lichtman, 1990). In contrast, extensive growth or retraction of individual NT branches has been observed at frog NMJs in vivo (Herrera et al., 1990; Chen et al., 1991; Ko, 1991; Langenfeld-Oster et al., 1993). These results confirm the structural remodeling inferred previously from histological staining of fixed frog muscles (Wernig et al., 1980). Thus, repeated in vivo observations at identified frog NMJs are a useful approach to examine the mechanisms of synaptic remodeling in the adult animal.

Because the synaptic extracellular matrix (ECM) is believed to play a role in axonal outgrowth and synapse formation (Sanes, 1989; Reichardt and Tomaselli, 1991), we have exam-

Received Sept. 25, 1995; revised Dec. 5, 1995; accepted Dec. 7, 1995.

This work was supported by National Institutes of Health Grant NS17954. We thank Drs. S. H. Astrow and A. Y. Chiu for critical comments on this manuscript, and Ms. Alicia Thompson for technical assistance.

Correspondence should be addressed to Dr. Chien-Ping Ko at the above address. Copyright (C) 1996 Society for Neuroscience $0270-6474 / 96 / 161780-11 \$ 05.00 / 0$ ined the involvement of synaptic ECM in the remodeling of NTs at living frog NMJs. By double labeling NTs with 4-di-2Asp (Magrassi et al., 1987) and synaptic ECM with rhodamineconjugated peanut agglutinin (PNA) (Ko, 1987), we have demonstrated dramatic remodeling in both NTs and synaptic ECM at frog NMJs in normal muscles (Chen et al., 1991; Ko, 1991) and in muscles in which nerve sprouting was induced (Chen and Ko, 1994). In addition, synaptic ECM longer than NT branches, sometime by tens of microns, was seen at NMJs that showed growth and sprouting. These observations led us to propose a hypothesis that synaptic ECM extends before, and may play a role in, the growth of the NT during synaptic remodeling in the adult.

Although the result of the repeated in vivo observations was confirmed with histological staining in our previous studies, light microscopy might not detect fine NT sprouts at the extended, synaptic ECM region. Furthermore, it is unknown whether Schwann cells may lead in NT sprouting at the extended region. Because PNA stains predominantly the ECM around Schwann cells at the NMJ (Ko, 1987), the extended PNA staining may be indicative of Schwann cell processes preceding the NT growth. The present study aimed to use electron microscopy in combination with repeated in vivo observations to examine the mechanism of synaptic remodeling. With this novel approach, we have found results consistent with the hypothesis that synaptic ECM is longer than the growing NT and that Schwann cells also may lead the NT outgrowth during synaptic remodeling at adult frog NMJs. Preliminary results of this work have been reported previously (Ko et al., 1992). 


\section{MATERIALS AND METHODS}

Repeated in vivo observations. The procedure for repeated in vivo observations of identified NMJs is identical to that described previously (Chen et al., 1991). Experiments were performed on adult frogs (Rana pipiens) of similar size (rump-to-nose length, $7-8 \mathrm{~cm}$ ) and weight (30-41 gm). To stain NMJs in vivo, frogs were anesthetized by immersion in $0.2 \%$ tricaine methanesulfonate (Sigma, St. Louis, MO) for $\sim 30 \mathrm{~min}$ and then kept on ice to maintain a low body temperature throughout the surgery and staining. An incision $(2-3 \mathrm{~cm})$ was made through the skin of the right leg to expose the sartorius muscle. NMJs were double labeled with a fluorescent dye, 4-(4- diethylamino-styryl)- $N$-methylpyridinium iodide (4-di2-Asp, $10 \mu \mathrm{M}$ in Ringer's solution, $3 \mathrm{~min}$ ) for NTs (Magrassi et al., 1987) and tetramethylrhodamine (TMR) isothiocyanate-conjugated PNA (50 $\mu \mathrm{g} / \mathrm{ml}$ in Ringer's solution, $30 \mathrm{~min}$ ) for the synaptic ECM (Ko, 1987). The stained muscle was rinsed with Ringer's solution containing (in $\mathrm{mm}$ ): $\mathrm{NaCl} 111, \mathrm{KCl} 2, \mathrm{CaCl}_{2} 1.8$, HEPES 5, pH 7.2, for 5-10 min. The frog then was placed on a cold $\left(0-4^{\circ} \mathrm{C}\right)$ metal plate attached to the stage of an epifluorescence microscope.

The stained NMJs were visualized with a $50 \times$ water-immersion objective (numerical aperture 1.0, Leica, Deerfield, IL). To identify individual NMJs for repeated observations, landmarks such as the myelinated nerve and blood vessels were noted. Filters for blue excitation-green emission (BP490/EY475, G520, Olympus, Lake Success, NY) were used to visualize 4-di-2-Asp-stained NTs, and filters for green excitation-red emission (BP545/EY550, R610, Olympus) were used for TMR-PNA-stained ECM. To minimize potential adverse effects of excessive illumination (Herrera and Banner, 1990), a small aperture and a $1.2 \%$ transmittance neutral density filter were used to attenuate the light source (100 W mercury), and the total exposure time for viewing both fluorescent labels was kept to $<20 \mathrm{sec} / \mathrm{NMJ}$. The images were captured by a silicon-intensified target camera (SIT 66, Dage, Michigan City, IN) and processed by a computer. A digital image processor (Image-1, Universal Imaging, West Chester, PA) was used for averaging 64 frames and processing to reduce background and enhance contrast. The processed images then were stored on videotape and/or computer diskettes and produced using a video printer (UP-850N, Sony) or a $35 \mathrm{~mm}$ camera photographing the videomonitor.

After the in vivo observations, two $2-3 \mathrm{~mm}$ segments of the contralat- eral sciatic nerve were removed and placed underneath the observed muscle as described previously (Chen and Ko, 1994). Nerve segments can induce extensive NT sprouting in about $\sim 2-3$ months (Diaz and PecotDechavassine, 1990; Chen and Ko, 1994). After implantation of the nerve segments, the skin incision was closed with sutures and the animal allowed to recover. Two to three months later, the right sartorius muscle was restained with 4-di-2-Asp and TMR-PNA, and the NMJs identified previously were viewed and recorded a second time. Some NMJs were viewed a third time. The subsequent viewing procedures and image processing were the same as the first observations as described above.

Electron microscopy of identified neuromuscular junctions. After the final observation in vivo, the frog was killed and the right sartorius muscle was fixed in situ for $1 \mathrm{hr}$ at room temperature by covering with a piece of Kimwipe soaked with $2 \%$ glutaraldehyde in frog Ringer's solution. The muscle was rinsed briefly with Ringer's solution and then dissected out and incubated with horseradish peroxidase (HRP)-conjugated PNA (100 $\mu \mathrm{g} / \mathrm{ml}$ in Ringer's solution) for $1 \mathrm{hr}$ at room temperature, or at $4^{\circ} \mathrm{C}$ overnight. After the incubation, the muscle was rinsed three times with Ringer's solution for $15 \mathrm{~min}$ each and subjected to the peroxidase enzyme reaction using a peroxidase substrate kit (Vector, Burlingame, CA). The identified NMJs were photographed through a compound microscope, and the muscle was post-fixed overnight with $2 \%$ glutaraldehyde at $4{ }^{\circ} \mathrm{C}$.

Before preparation for thin-section electron microscopy, the fluorescent images of the in vivo observations of the identified NMJs were compared with the HRP-PNA staining of the same muscles to locate the identified NMJs in the fixed muscle. Only the NMJs that showed synaptic ECM longer than the NT were prepared for electron microscopy. The muscle fiber containing the identified NMJ and a few neighboring fibers and their nerve supply were teased from the rest of the muscle. The tissue was fixed further for $1 \mathrm{hr}$ with $1 \%$ osmium tetroxide, then dehydrated with an ethanol series and flat embedded with Poly/Bed 812 (Polysciences, Warrington, PA). The identified NMJ observed through the thin $(0.1-0.5 \mathrm{~mm})$ wafer of plastic under a dissection microscope was trimmed carefully with a razor blade to retain primarily the region with synaptic ECM longer than the NT. The trimmed block was photographed through a compound microscope, and the distance between the edge of the razor blade trimming to be sectioned with a diamond knife and several land-
N T
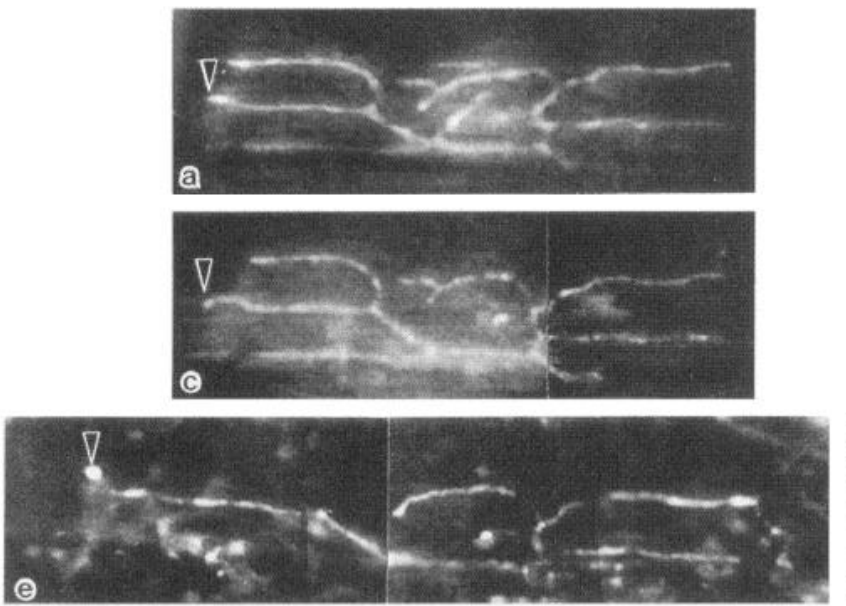

PNA
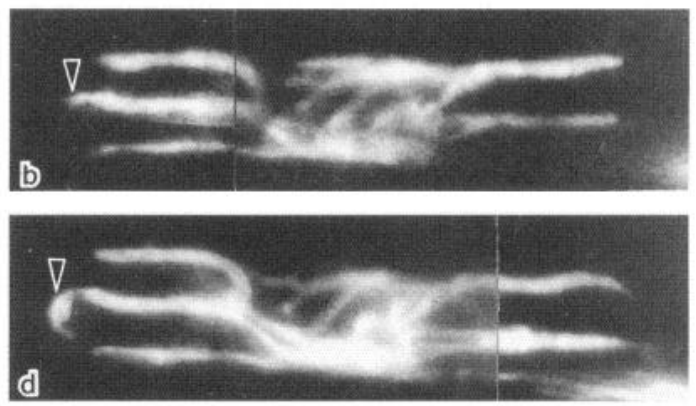
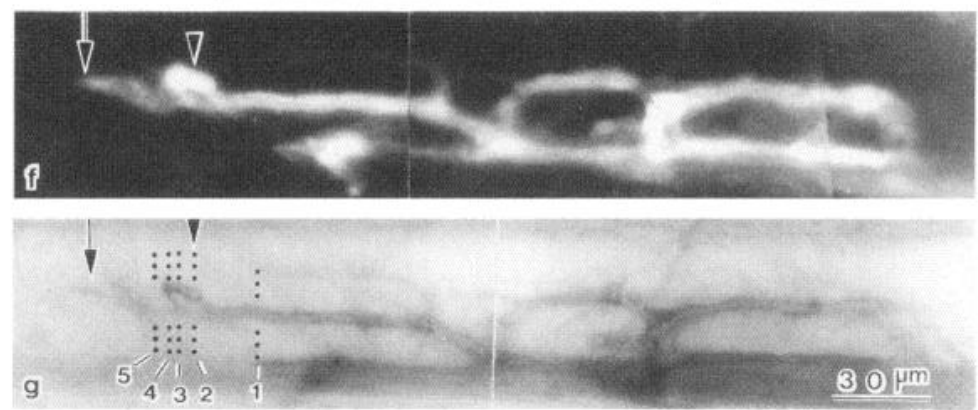

Figure 1. An NMJ, double labeled with 4-di-2-Asp for the $N T(a, c, e)$ and rhodamine-tagged $P N A(b, d, f)$ for synaptic ECM, was observed in vivo with video-enhanced fluorescence microscopy at three different dates, followed by staining with HRP-conjugated PNA $(g)$. NT sprouting was induced by a nerve graft immediately after the first observation. $a, b$, First observation showed a good match between the NT and PNA staining at the branch identified by arrowheads. $c, d$, Second observation, made $68 \mathrm{~d}$ later, showed a slight extension of this branch, which had PNA staining (arrowhead in $d$ ) longer than the NT (arrowhead in $c$ ).e, $f$, Third observation, made $75 \mathrm{~d}$ after the second observation, revealed a more drastic extension of this branch with synaptic ECM (arrow in $f$ ) longer than the NT (arrowhead in $e$ ). This extended, PNA-stained region is indicated between the arrow and arrowhead in $f$. $g$, After the third observation, the same junction was stained with HRP-PNA. Arrow and arrowhead indicate the same positions as those in $f$. The numbers indicate the positions of the cross-sections shown in the electron micrographs in Figure 2. Scale bar, $30 \mu \mathrm{m}$ for all panels. 

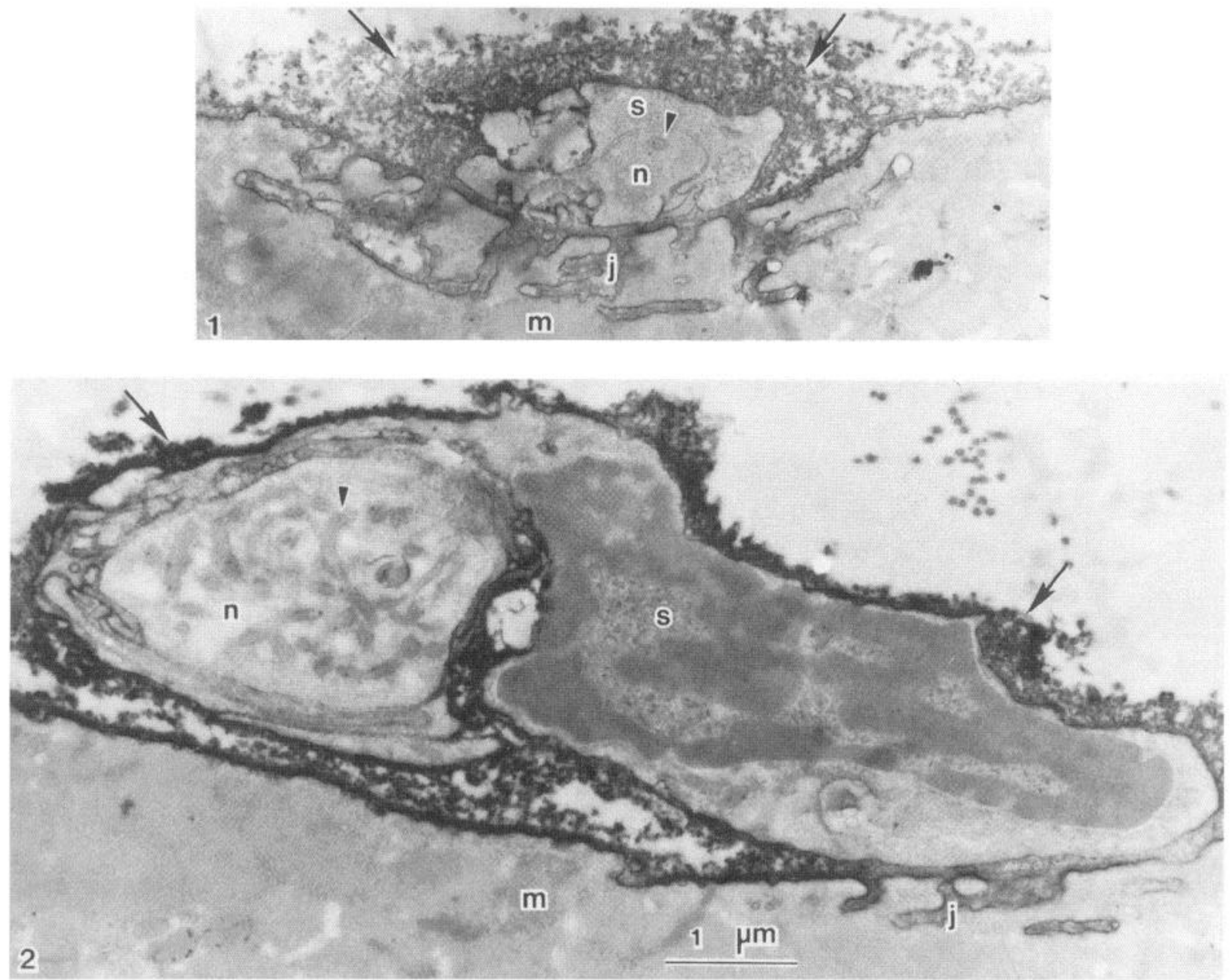

Figure 2. Electron micrographs of cross-sections taken at five different positions of the NMJ shown in Figure $1 g$. So as not to mask the HRP reaction product, grid staining was not used in these sections. The electron micrographs in this figure were enlarged to the same final magnification. 1 , Typical morphology of a normal NMJ with HRP reaction product (arrows) in the ECM surrounding the Schwann cell $(s)$ and in the junctional folds $(j)$ of the muscle fiber $(m)$. The arrowhead indicates mitochondria in the NT $(n)$. The abbreviations apply to all of the electron micrographs. 2, This position, corresponding to the tip of the NT as shown in Figure 1e, reveals a large nerve ending packed with numerous mitochondria (arrowhead). (Figure continues)

marks, such as the branches of NT, end of the terminal, and synaptic ECM staining, were measured. This information allowed confirmation of the location of the region observed in thin sections. The trimmed block was glued onto a resin stud for semiserial thin sections. Silver-gray thin sections $(\sim 80 \mathrm{~nm})$ were collected onto slot grids coated with formvar. By counting the number of thin sections, correlation between light and electron microscopy could be made. All thin sections were examined without the conventional grid staining of lead citrate and uranyl acetate. Although the omission of the grid staining reduces the contrast of the cell structures, it allows a better detection of HRP reaction product under a transmission electron microscope.

\section{RESULTS}

\section{Extension of synaptic ECM and Schwann cell processes beyond the NT}

\section{Light microscopy}

Repeated in vivo observations of identified NMJs double stained with 4-di-2-Asp (Magrassi et al., 1987) for NT and TMR-PNA (Ko, 1987) for synaptic ECM were performed before electron microscopy. The NMJs showing synaptic ECM longer than the NT at the final in vivo observations were selected for additional study with electron microscopy. In a total of nine identified NMJs in seven frogs that were studied by combining light and electron microscopy, seven NMJs were processed successfully and reiden- tified with electron microscopy. In these seven NMJs, five showed extension of a junctional branch, whereas two showed retraction. Figure 1 shows an example of light microscopic observation of an NMJ with synaptic ECM extended longer than the NT that was examined later with electron microscopy (Fig. 2). At the initial observation, the length of the NT (Fig. 1a) matched that of the synaptic ECM revealed by PNA staining (Fig. $1 b$ ) as exemplified by the branch indicated by arrowheads. Immediately after this initial observation, segments of contralateral sciatic nerve were placed on the muscle surface to induce nerve sprouting (Diaz and Pecot-Dechavassine, 1990; Chen and Ko, 1994). At the second observation of the same NMJ made $68 \mathrm{~d}$ later, the length of the NT branch indicated by the arrowhead (Fig. 1c) showed little change. However, synaptic ECM extended beyond the NT by $\sim 13$ $\mu \mathrm{m}$ (Fig. 1d). The same branch, observed a third time ( $75 \mathrm{~d}$ after the second observation), showed a more drastic extension in both NT (Fig. 1e) and PNA-staining (Fig. 1f). Whereas the NT elongated by $\sim 35 \mu \mathrm{m}$ (arrowhead in Fig. 1e), synaptic ECM extended even more, by $58 \mu \mathrm{m}$ (arrow in Fig. 1f), in comparison with the second observation. Thus, at the third observation, synaptic ECM extended past the NT by a total of $36 \mu \mathrm{m}(58+13-35=36)$ as shown in Figure $1 f$ (region between the arrow and arrowhead). It 

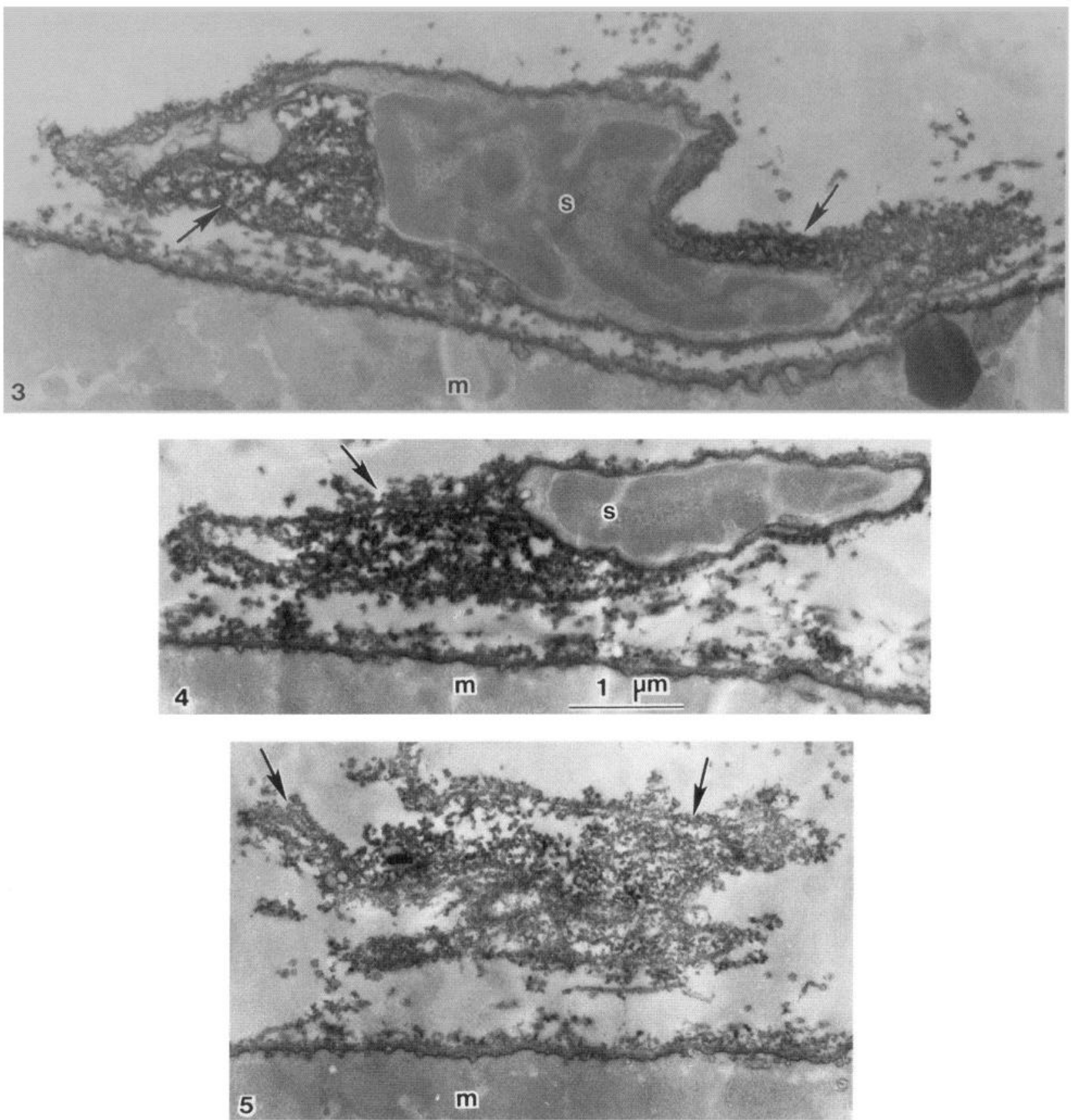

Around the NT is a large Schwann cell surrounded by PNA-stained ECM (arrow). 3, This position, $5 \mu$ m distal to position 2, shows the Schwann cell with PNA-stained ECM but without a discernible NT. 4, This position is $2 \mu \mathrm{m}$ distal to position 3 and still reveals the Schwann cell (although smaller) and PNA-stained ECM, which is somewhat separated from the muscle surface. Note the absence of junctional folds distal to this position. 5, Neither NT nor Schwann cells are present at this position, $3 \mu \mathrm{m}$ distal to position 4. Only PNA-stained ECM, which is even farther away from the muscle surface, is seen at this position.

also is noted that, although this branch extended, two other branches of the same junction retracted (Fig. 1e). Because it was of interest to study the extension of synaptic ECM extension before NT sprouting, we focused on the extending branch of this particular junction. The relationship between synaptic ECM and retracting NT was examined at other junctions as described later in Figures 5 and 6.

After the final in vivo observation, the same NMJ was stained with HRP-PNA. The staining pattern of HRP-PNA (Fig. $1 g$ ) was identical to that of TMR-PNA (Fig. 1f). Thus, $50 \mu \mathrm{g} / \mathrm{ml}$ TMR-
PNA did not saturate all of the PNA-binding sites, and some were available for subsequent staining with HRP-PNA. As notes in the previous report (Ko, 1987), HRP-PNA outlines the NT and showed a "railroad-track" staining pattern. After the NMJ stained with HRP-PNA was photographed, the single muscle fiber identified and a few neighboring fibers were teased apart from the rest of the muscle fibers, then flat-embedded and processed for electron microscopy as described in Materials and Methods. The numbered locations, shown in Figure $1 g$, indicate the positions where micrographs were subsequently taken (Fig. 2). 


\section{Electron microscopy}

At position 1, where the NT and synaptic ECM were both revealed by light microscopy, a typical cross-sectional view of normal NMJ was seen with electron microscopy (Fig. 2-1). The nerve ending $(n)$ and Schwann cell $(s)$ were covered with HRP-PNA staining reaction product (arrows) as shown previously (Ko, 1987, 1991). The synaptic ECM stain also was observed in the junctional folds $(j)$ of the muscle fiber $(m)$. As shown at normal NMJs (Engel, 1994), the elaborated junctional folds were not restricted to the region directly underneath the NT. Junctional folds also were seen at the area contacted by Schwann cell processes and the region slightly beyond the junctional gutter. Position 2 is located at the tip of the NT as shown with light microscopy (arrowheads in Fig. $1 e-g$ ). Note that the tip of the NT had larger and brighter 4-di-2-Asp staining when compared with the rest of the N'I (Fig. $1 e$ ). As seen at position 1, PNA also outlined the NT at this region (arrowheads in Fig. 1f $g$ ). Figure 2-2 shows a cross-sectional view of this position with the magnification identical to that at position 1 (Fig. 2-1). The size of the NT $(n)$ is much larger than that at position 1, as one might expect from the light microscopic observation. In addition, the enlarged nerve ending is full of numerous mitochondria (arrowhead in Fig. 2-2). This result may explain the brighter fluorescent staining at the tip of the nerve ending with 4-di-2-Asp, which is thought to label mitochondria (Magrassi et el., 1987). In addition to showing the nerve ending, the crossscction shows a large Schwann cell (s) with a prominent nucleus that occupies a large surface including the area with a few junctional folds $(j)$. Similar to junctional folds extending beyond the region occupied by NT shown in Figure 2-1, these few junctional folds probably are extended from the nearby junctional gutter (proximal to position 2) that already has been occupied by NT. This electron microscopic result confirms the previous suggestion (Ko, 1987; Chen et al., 1991) that PNA staining is capable of revealing the location of the Schwann cell body using light microscopy. As expected from the light microscopic staining, HRPPNA also stains the synaptic ECM around the Schwann cell and the junctional folds as shown in the electron micrograph.

Position 3 is $5 \mu \mathrm{m}$ distal to position 2 and, thus, is at the region where the synaptic ECM appears to extend beyond the NT as viewed with light microscopy. If 4-di-2-Asp is a reliable staining for the NT, one would expect to observe synaptic ECM but no NT at position 3 with electron microscopy. This is confirmed in Figure 2-3. The reaction product (arrows) of HRP-PNA was seen in the ECM around the cell containing a nucleus. Presumably, this is the Schwann cell, because the NT does not contain a nucleus. Note that at position 3 , the postsynaptic membrane was indented but did not have distinctive junctional folds as seen at positions 1 and 2. Furthermore, the distance between the Schwann cell membrane and the muscle membrane was wider at this position than that at the normal junctional site at positions 1 and 2. The wide separation between the Schwann cell and the muscle membrane at the sprouting junctional region also has been observed by Wernig et al. (1981a). An even wider separation $(\sim 1 \mu \mathrm{m})$ between the Schwann cell and the muscle membrane was seen at position 4 , which is $7 \mu \mathrm{m}$ distal to the end of the NT. At this position, there was neither indentation nor junctional folds in the muscle membrane $(m)$. Another interesting feature of this region was that the major area of HRP-PNA stained-ECM (arrow in Fig. 2-4) was seen at one side of the Schwann cell, but not at the region immediately on the Schwann cell membrane. Al position 5, which is $10 \mu \mathrm{m}$ distal to the end of the NT, the Schwann cell was absent.
However, HRP-PNA stained-ECM (arrows in Fig. 2-5) was still present. As at position 4, the ECM at position 5 was separated from the muscle membrane $(m)$, which did not have indentations or junctional folds. The structure between position 5 and the tip of PNA-stained ECM indicated by an arrow was similar to that seen at position 5 (data not shown).

\section{Extension of synaptic ECM, but not Schwann cell processes, longer than the NT}

Light microscopy

With findings similar to the above results, Figure 3 shows repeated observations of an NMJ with synaptic ECM longer than the NT after induced sprouting. At the first observation, the NT staining was correlated with PNA staining as exemplified by a branch indicated by arrowheads in Figure 3, $a$ and $b$. At the second observation $115 \mathrm{~d}$ later, the NT branch was elongated by $17 \mu \mathrm{m}$ as indicated by an arrowhead in Figure $3 c$. Note that some areas of the muscle fiber shown in Figure 3, a and $c$, were brighter because of higher background staining of the muscle fiber. At the second observation, PNA staining also revealed an extension of the ECM by $27 \mu \mathrm{m}$ compared with the first observation. Thus, the PNA-stained ECM was longer than the NT by $10 \mu \mathrm{m}$ (between arrow and arrowhead in Fig. $3 d$ ) at the second observation. Note that PNA staining appeared as a large diffuse mass at the tip of this branch. This type of staining pattern often is seen at newly formed junctional areas (Chen et al., 1991; Chen and Ko, 1994).

\section{Electron microscopy}

Electron micrographs of thin sections at three locations marked by dotted lines in Figure $3 d$ are shown in Figure 4 . At position 1, typical ultrastructure of the NMJ stained with HRP-PNA was seen. Both NT $(n)$ and Schwann cell $(s)$ occupied the NMJ with an indented junctional gutter (arrow) and junctional folds (j) (Fig. 4-1). Synaptic ECM, stained with IIRP-PNA (arrowhead) also was seen. The result is consistent with the light microscopic finding showing both NT and synaptic ECM at this position (Fig. $3 d$ ).

Position 2 corresponds to the tip of the NT revealed with 4-di-2-Asp staining at the second observation (arrowheads in Fig. $3 c, d)$. Electron microscopy of this position showed a NT $(n)$ enveloped by a cell that is, in turn, surrounded by PNA-stained ECM (arrowhead in Fig. 4-2). This cell presumably is the Schwann cell $(s)$, although without the appearance of a nucleus, the identity of this cell as a Schwann cell is not as certain as that seen in Figure 2. Position 2 shows only an indentation of junctional gutter (arrow) without the postjunctional folds.

Electron microscopy confirms the absence of NTs at position 3 (Fig. 4-3), which is only $1 \mu \mathrm{m}$ distal to position 2 . Similar to the above example, there are no postjunctional folds, although a slight indentation was seen at position 3. In addition, synaptic ECM stained with HRP-PNA extended $10 \mu \mathrm{m}$ past the NT as predicted from the light microscopic result. However, in contrast to Figure 2, which shows a Schwann cell extending beyond the NT, neither NT nor Schwann cells were present at this extended region (Fig. 4-3). The results exemplified by this junction were found in four of five junctions that showed extension of synaptic ECM longer than the NT. Thus, Schwann cells often do not appear extending longer than the NT at the region where synaptic ECM is found beyond the sprouting NT. 
N T
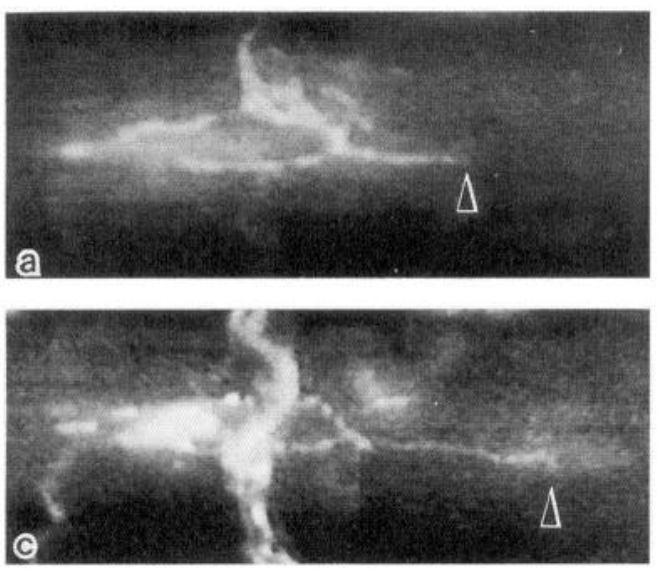

P N A
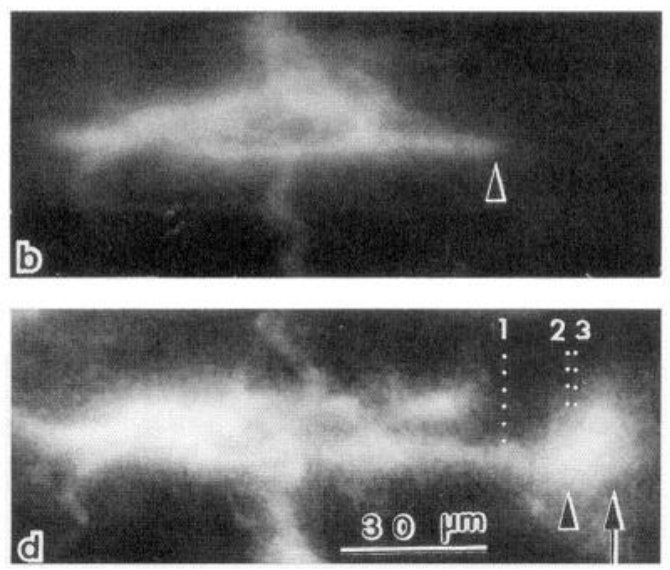

Figure 3. An NMJ double labeled with 4-di-2-Asp $(N T ; a, c)$ and rhodaminetagged PNA $(P N A ; b, d)$ showing extension of synaptic ECM longer than the NT. $a, b$, First observation showed a branch (arrowheads) with a precise alignment between NT and PNA staining. $c, d$, Second observation made $115 \mathrm{~d}$ after the first observation showed an elongation of $17 \mu \mathrm{m}$ at the NT branch indicated by an arrowhead. The same NMJ, however, revealed a $27 \mu \mathrm{m}$ extension of synaptic ECM at this branch with a blob of fuzzy PNA staining at the region ECM longer than the NT (between arrow and arrowhead in $d$ ). The numbers indicate the positions of the crosssections shown in the electron micrographs in Figure 4. The calibration bar applies to $a-d$.

\section{Persistence of junctional folds and synaptic ECM after retraction of NT}

\section{Light microscopy}

Retraction of NT sometime was observed as shown at an NMJ in Figure 5. At the initial observation, there was a good correlation between NT staining (Fig. $5 a$ ) and PNA staining (Fig. $5 b$ ), including the branch indicated by arrowheads. At the second observation $75 \mathrm{~d}$ later, although 4-di-2-Asp staining showed a dramatic extension of one NT branch (upper left region of the NMJ), there was retraction of a NT branch by $8 \mu \mathrm{m}$, as indicated by an arrowhead (compare arrowheads in $a$ and $c$ ). In contrast to the NT, there was a slight extension of synaptic ECM by $5 \mu \mathrm{m}$ at this junctional branch (compare arrowhead in $b$ with arrow in $d$ ). Thus, synaptic ECM was longer than the retracting NT branch by $13 \mu \mathrm{m}$ at this branch (compare arrow in $d$ with arrowhead in $d$ or $c$ ).

\section{Electron microscopy}

Figure 6-1 shows ultrastructure of the junctional branch corresponding to position 1 near the tip of the NT as seen with light microscopy (Fig. $5 d$ ). At this position, PNA-stained synaptic ECM (arrow) occupied the junctional gutter with elaborated junctional folds. However, the junctional area was only partially occupied by a small NT $(n)$ wrapped by Schwann cell processes $(s)$. The partial occupation by the NT is shown further in a neighboring section (Fig. 6-2), $3 \mu \mathrm{m}$ away, corresponding to position 2 located at the tip of the NT branch (Fig. $5 c$ ). At this position, only a small remnant of NT surrounded by Schwann cell processes is seen. At position 3 , only $1 \mu \mathrm{m}$ from position 2 , neither NT nor Schwann cell was present (Fig. 6-3). However, synaptic ECM occupied this "empty" junctional gutter, where conspicuous postjunctional folds were shown. This empty gutter with ECM stained by PNA still was seen, $8 \mu \mathrm{m}$ distal to position 3, near the tip of PNA-stained ECM at position 4 (Fig. 6-4). The presence of junctional folds is consistent with the light microscopic observation suggesting that the mismatch between NT and synaptic ECM staining resulted from retraction of NT at this junction. This finding is seen at the two junctions that showed retraction in the present study. The result contrasts with that seen in the five extending junctions that showed no junctional folds at the extended PNA-stained junctional region.

\section{DISCUSSION}

We have demonstrated the remodeling of synaptic ECM in relation to NT sprouting at identified frog NMJs with repeated in vivo observations followed by electron microscopy. This combined light and electron microscopic approach presents not only the dynamic history of identified junctional branches but also ultrastructural information about the site of synaptic remodeling. The new findings support the hypothesis that extension of synaptic ECM precedes, and may play a role in, the NT outgrowth in the following manner: First, synaptic ECM extending beyond the growing NT was confirmed with electron microscopy. In addition, electron microscopy has confirmed the absence of junctional folds at the region where synaptic ECM extended beyond NTs. In contrast, elaborated junctional folds were seen at the junctional branch that retracted. Finally, at the extended synaptic ECM region, Schwann cell processes longer than the NT were observed. Thus, Schwann cells also may play a role in NT sprouting.

A possible sequence of synaptic remodeling based on the present report and our previous findings (Chen at al., 1991; Ko, 1991; Chen and Ko, 1994) is proposed (Fig. 7). Before junctional extension or retraction, the NT, Schwann cell, and synaptic ECM all are colocalized at the NMJ $(A)$. In response to unknown sprouting signals, a diffuse mass of synaptic ECM extends in front of the NT and Schwann cell $(B)$. The ECM could be secreted by the muscle fiber and/or the Schwann cell. Following this recently deposited synaptic ECM, a Schwann cell process extends in front of NT $(C)$. The extension of the Schwann cell is followed by an elongation of the NT $(D)$. The above sequence of $C$ and $D$ is repeated until both the NT and Schwann cell match the preceding synaptic ECM $(E)$. Once the sprouting is completed, junctional folds are induced at the new site. In contrast to the nerve extension, if and when retraction of the NT and Schwann cell occurs, junctional folds and synaptic ECM are left behind in the empty gutter $(F)$. Eventually, synaptic ECM and junctional folds also disappear after long-term denervation at the empty gutter $(G)$.

\section{The reliability of NT staining with 4-di-2-Asp}

The mitochondria dye 4-di-2-Asp has been used widely to reveal NTs in vivo. However, a common concern is whether NTs, especially at the growing tip, can be revealed faithfully with 4-di-2-Asp. Although the 4-di-2-Asp staining of NTs has been confirmed by histological staining at frog NMJs (Herrera and Banner, 1990; Herrera et al., 1990; Chen et al., 1991; Chen and Ko, 1994), the possibility of smaller nerve sprouts present at the growing tip but below the resolution of light microscopy could not be excluded in those studies. The present study using electron microscopy has 


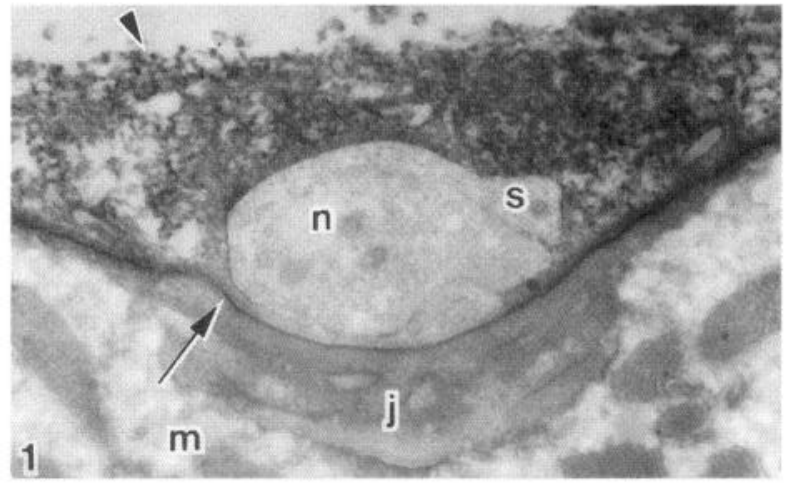

Figure 4. Electron micrographs of cross-sections taken at three different positions of the same NMJ as indicated in Figure 3. 1, Typical ultrastructure of a normal NMJ with the NT $(n)$ and Schwann cell $(s)$ occupying the indented junctional gutter (arrow) of a muscle fiber $(\mathrm{m})$. Consistent with the fluorescence microscopic observation shown in Figure 3, synaptic ECM labeled with HRP-PNA (arrowhead) is seen at this position. 2, This position, corresponding to the tip of the NT (arrowhead in Fig. $3 c, d$ ), reveals a NT and a cell (presumably the Schwann cell) ( $s$ ) surrounded by synaptic ECM stained with HRP-PNA (arrowhead). The arrow points to a slight indentation of the junctional gutter without junctional folds at this position. 3 , This position is only $1 \mu \mathrm{m}$ distal to position 2. As predicted from the light microscopic observation (Fig. 3), NT is absent from this position where PNA-stained ECM (arrowhead) is seen. In contrast to the NMJ shown in Figure 2, the Schwann cell also is absent from this region. Although a slight indentation (arrow) is observed, no elaborate junctional folds $(j)$ as seen at position 1 are shown at positions 2 and 3 . The calibration applies to the electron micrographs of all positions.
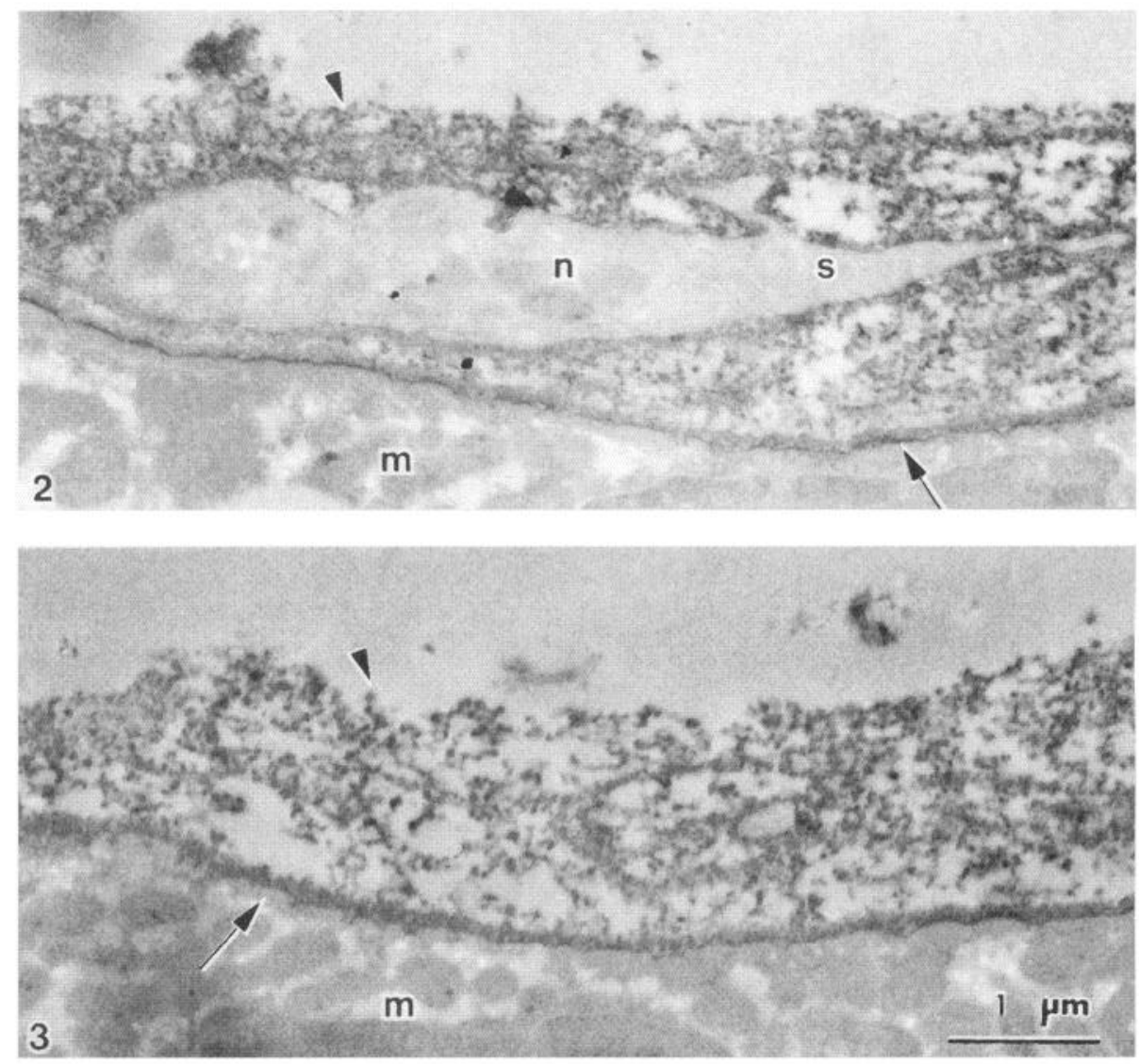

excluded this possibility. Thus, the present result contributes an important validation of previous studies in which 4-di-2-Asp was presumed to stain the entire NT and provides additional support for our hypothesis that synaptic ECM extends longer than the growing NT.

\section{The dynamic relationship between synaptic ECM and NTs}

Synaptic ECM longer than the NT has been seen at junctional branches that extend, as well as at those that retract, during the interval of the repeated in vivo observations (Chen et al., 1991; Chen and Ko, 1994). Thus, one concern regarding the interpretation of these results is whether the mismatch between the NT and synaptic ECM at a particular junctional branch is attributable to a retraction of the NT preceding ECM removal or attributable to an extension of ECM preceding NT growth. To distinguish between these two possibilities, we combined repeated in vivo observations with histological staining in these previous studies.
We found junctional folds revealed by acetylcholinesterase (AChE) staining in the junctional region that showed detraction, but neither AChE nor junctional folds in the region of the branch that displayed extension with synaptic ECM longer than the sprouting NT. In the present study, we used electron microscopy to extend these observations. The contrast in the appearance of junctional folds is consistent with the interpretation on extension versus retraction based on the repeated in vivo observations. The present results support further the previous interpretation of static images from histological studies that suggested that empty synaptic gutters with $\mathrm{AChE}$ and junctional folds but without overlying NTs were attributable to nerve retraction (Wernig et al., 1980, 1981a,b). In contrast, junctional folds were either missing or less well developed in the region where Schwann cells enclosed bulbous NTs suspected to be sprouting (Wernig et al., 1981a; Anzil et al., 1984). Thus, the absence or presence of junctional folds and AChE may be used as one criterion to interpret whether 

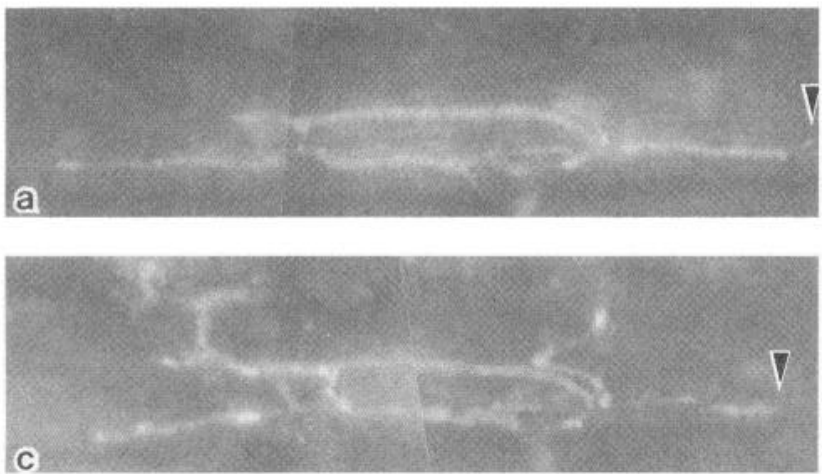

P N A
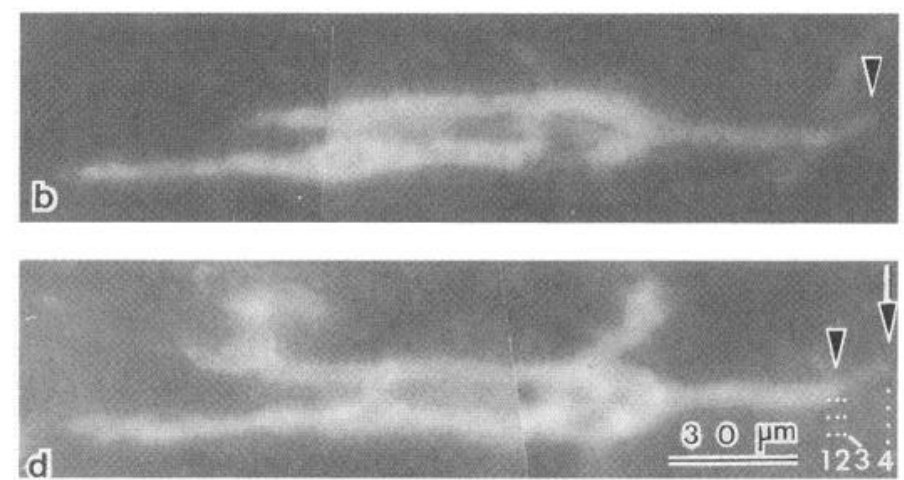

Figure 5. An NMJ double labeled with 4-di-2-Asp $(N T ; a, c)$ and rhodamine-PNA $(P N A ; b, d)$ showing retraction of the NT before synaptic ECM. $a$, $b$, First observation showed a precise alignment between the NT and synaptic ECM at a branch (arrowheads). $c, d$, Second observation revealed a retraction of NT by $8 \mu \mathrm{m}$ at this branch (arrowhead in $c$ ), whereas synaptic ECM stained by PNA at this branch remained. The region between the arrow and arrowhead in $d$ probably represents an "empty" junctional area, as demonstrated further in the electron micrographs in Figure 6. The numbers in $d$ indicate the positions of the cross-sections shown in the electron micrographs in Figure 6. Scale bar, $30 \mu \mathrm{m}$ for all panels.

the junctional region is undergoing extension or retraction, respectively (compare Fig. $7 B$ with $F$ ).

Although the absence of junctional folds and AChE per se is not unequivocal evidence to prove that the junctional region is undergoing extension, our previous studies have shown other evidence consistent with the hypothesis that synaptic ECM leads the NT outgrowth (Chen et al., 1991; Ko, 1991; Chen and Ko, 1994). For example, we have seen many examples of junctions with continual extension of synaptic ECM without any changes in the NT length in successive observations. If this mismatch is attributable to retraction of the NT, one would have to assume that the NT could extend tens or even hundreds of microns and induce rapidly only PNA-stained ECM, then retract rapidly, before $\mathrm{AChE}$ and junctional folds are formed, to exactly the same points at various times of observation. Although this possibility cannot be excluded by the present technique, it seems highly unlikely that we would catch these kind of images so often [Figs. 6, 7 in Chen et al. (1991); Fig. 5 in Ko (1991); Figs. 3, 6-8, 10 in Chen and Ko (1994)], given the slow processes of nerve sprouting in the frog. Alternatively, it is more likely that synaptic ECM appears before NT extension. This is supported further by our previous results showing junctions with synaptic ECM longer than the NT, even at the first observation. The NT then extends along the preceding synaptic ECM in the subsequent observations. Moreover, as predicted by our hypothesis, the percentage of NMJs showing synaptic ECM longer than the NT is increased greatly from $10 \%$ in normal NMJs to $65 \%$ in muscles in which nerve sprouting is induced.

\section{Do Schwann cells lead in NT sprouting?}

The present study has shown Schwann cell processes longer than the NT at a junctional region that was observed to be extending with repeated in vivo observations. This result strengthens the interpretation of previous electron microscopic studies that showed Schwann cell processes extended several microns in front of NTs presumed to be extending based on the static images from fixed tissues (Wernig et al., 1981a; Anzil et al., 1984). These studies suggest that Schwann cells also may lead and play a role in nerve sprouting. This idea is consistent with recent work suggesting that Schwann cells induce and guide axonal sprouting in mammalian muscles. Son and
Thompson (1995b) have shown that experimental manipulations that induce NT sprouting also induced terminal Schwann cells to extend processes. In addition, Schwann cell processes are associated with every nerve sprout and, in some cases, are longer than the nerve sprouts. The sprouts then may catch up with the preceding Schwann cell process. Thus, depending on the timing of the observation, one may not always find Schwann cell processes longer than the NT (compare Fig. $7 C$ with $D$ ). Consistent with the idea that Schwann cells lead NT growth, NMJs with NTs longer than Schwann cell processes or PNAstained ECM never were observed in the present study.

Extension of elaborate Schwann cell processes after denervation at NMJs has been demonstrated (Reynolds and Woolf, 1992; Astrow et al., 1994; Son and Thompson, 1995a,b). In addition, fibroblasts that proliferate after denervation produce growthpromoting ECM at the NMJ (Connor and McMahan, 1987; Gatchalian et al., 1989). Thus, both Schwann cells and ECM are capable of growing in the absence of the NT. However, it is unknown what triggers the extension of Schwann cells and the synaptic ECM in front of the sprouting NT. Van Mier and Lichtman (1994) have suggested that regenerating muscle fibers emit a signal that induces sprouting from nearby undamaged NTs. It is possible that the muscle fiber itself may produce ECM molecules and/or soluble factors that may induce the growth of Schwann cells and nerve sprouts. Alternatively, the Schwann cell itself can secrete ECM molecules (Schachner, 1990; Chiu et al., 1991; Bunge, 1994). Thus, it is possible that Schwann cells release ECM molecules that may, in turn, induce extension of Schwann cell processes and NT outgrowth during synaptic remodeling. The present observation showing PNA-stained ECM longer than Schwann cell processes is consistent with this idea. The idea also may explain the diffuse blobs of PNA staining often observed in front of the NT growth (Fig. 3) (also see Chen et al., 1991; Chen and Ko, 1994) and patches of ECM separated from the muscle surface (Fig. 2). This indistinct blob of synaptic ECM may become more defined in appearance as Schwann cell processes extend to the preceding ECM region. The blob of ECM without Schwann cell processes eventually would disappear, because the maintenance of PNA-stained ECM may depend on the Schwann cell (Somasekhar and Ko, 1991). It also is conceivable that during this 

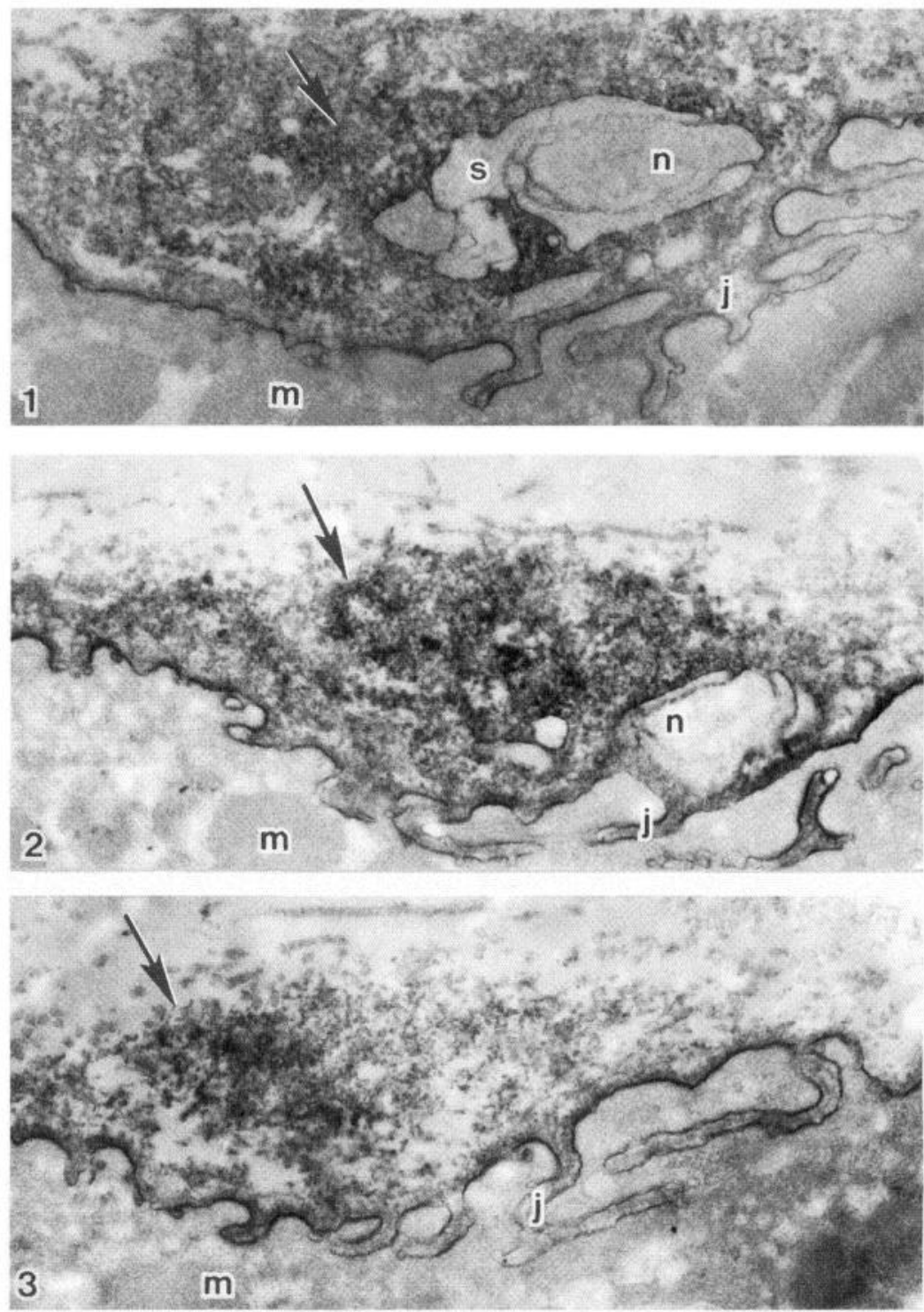

Figure 6. Electron micrographs of cross-sections taken at four different positions of the same NMJ as indicated in Figure 5. 1, At this position, $3 \mu \mathrm{m}$ proximal to the tip of the NT, the NT $(n)$ wrapped by the Schwann cell $(s)$ partially occupied the junctional area with HRP-PNA reaction product present in the conspicuous gutter indentation and junctional folds $(j) .2$, This position, corresponding to the tip of the NT, showed a small NT $(n)$ surrounded by Schwann cell processes and a large empty gutter filled with the reaction product of HRP-PNA (arrow). 3, At this position, $1 \mu \mathrm{m}$ distal to position 2, neither the NT nor Schwann cell was present as predicted from the light microscopic observation. In contrast to the extending junctional branch shown in Figures 2 and 4, extensive junctional folds $(j)$ and prominent indentation of the junctional gutter filled with PNA-stained ECM are seen at this branch showing retracting NT. 4, This position, corresponding to the very tip of PNA-stained ECM, showed HRP reaction product (arrow) without the NT and Schwann cells. Although elaborate junctional folds were observed distal to position 3 , only an indented junctional gutter was seen at position 4 . Scale bar, 1 $\mu \mathrm{m}$ for all panels.

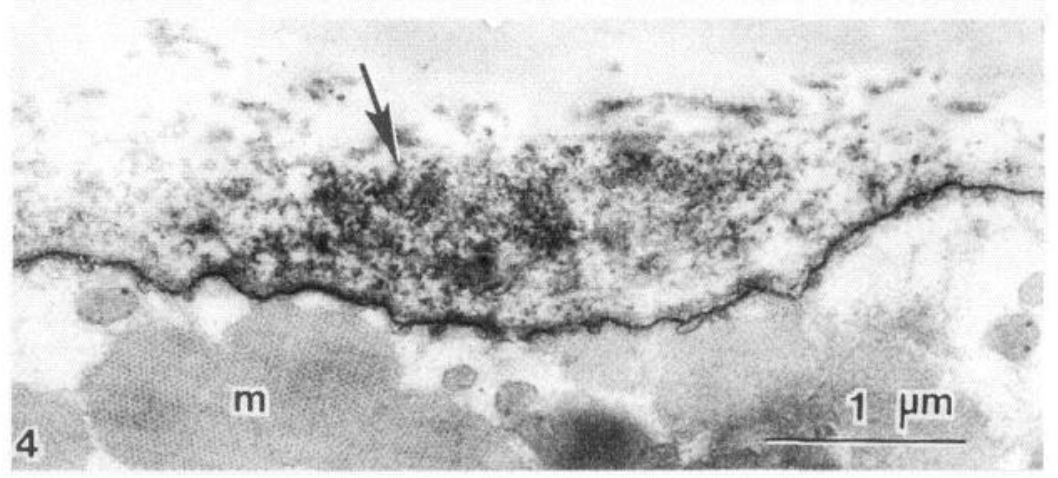

dynamic period, Schwann cell processes could release new ECM at a different site. This may explain the change in the direction of synaptic ECM extension as shown in Figure $1, d$ and $f$. However, the dynamic relationship between synaptic ECM and Schwann cell processes is not definite from the present study. An alternative explanation for synaptic ECM longer than Schwann cell processes could be that both are colocalized during the initial extension, but Schwann cell processes then may retract and leave behind the synaptic ECM. Another possibility is that a few small, thin Schwann cell processes may be present at the extended region but are indistinguishable from the meshwork of the HRP reaction product. Although we cannot rule out this possibility, the present result showing that HRP-PNA-stained ECM is the predominant occupant of the extended region is consistent with the suggestion that the ECM plays a key role in NT growth. To address the above two alternative explanations, one would need a specific marker for the terminal Schwann cell membrane with which to examine the dynamic relationship 


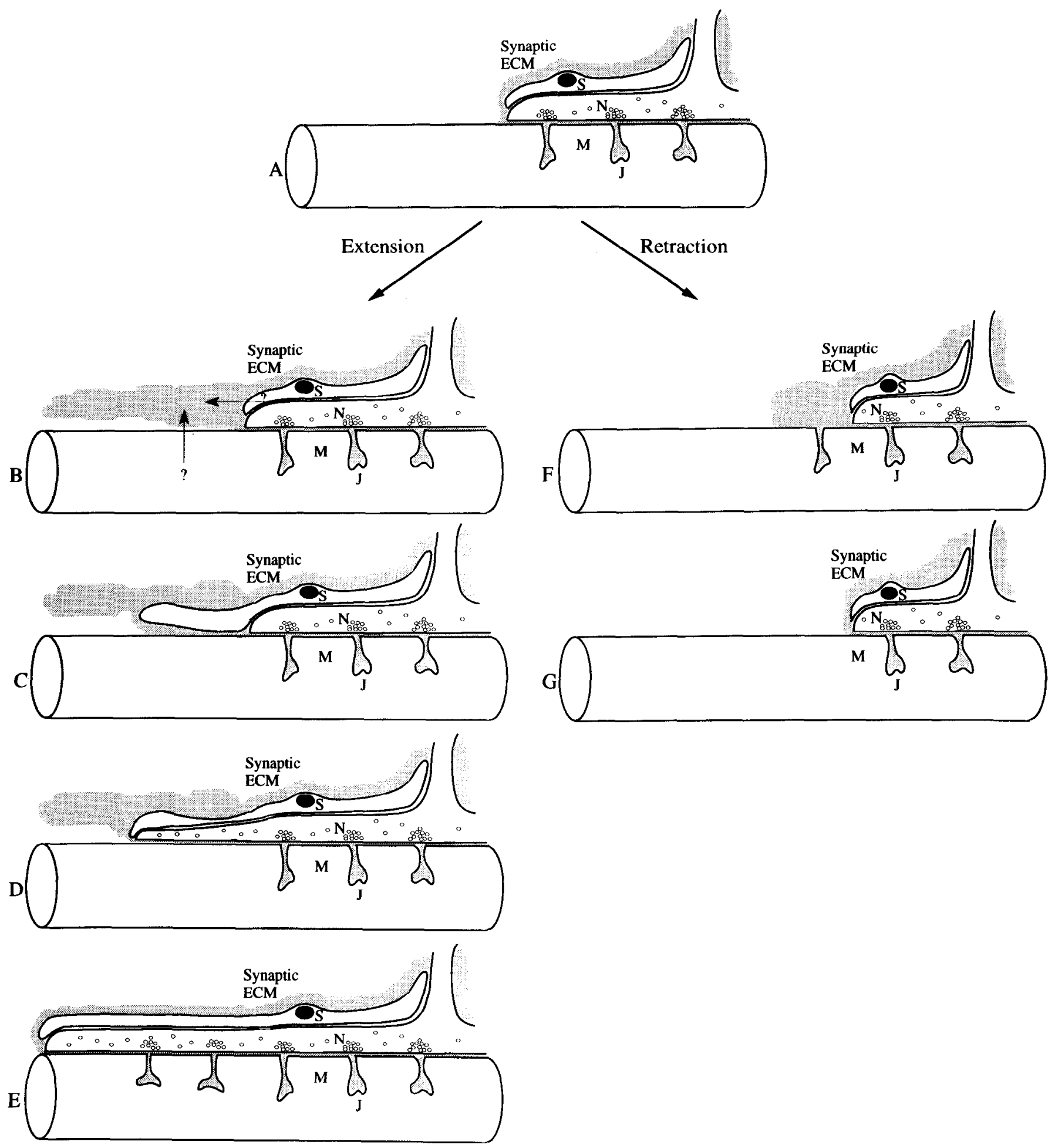

Figure 7. A schematic diagram showing a possible sequence of synaptic extension $(A-E)$ and retraction $(A, F, G)$ at the frog NMJ. $A$, A typical skeletal muscle fiber $(M)$ with the NT $(N)$, Schwann cell $(S)$, and synaptic ECM (stained with PNA; shaded area) colocalized at the NMJ, which contains junctional folds $(J) . B$, The first sign of synaptic sprouting is the extension of synaptic ECM in front of the NT and Schwann cell. The source of synaptic ECM is unknown and could be produced by muscle fibers and/or Schwann cells. $C$, Schwann cell processes follow the preceding synaptic ECM and lead the NT growth. $D$, The NT elongates and catches up with the preceding Schwann cell processes. $E$, Eventually, both the Schwann cell and the NT match the synaptic ECM after repeated sequence of $C$ and $D$. In addition, new junctional folds are formed at the sprouting region, and this completes the extension of a junctional branch. $F$, The NT and Schwann cell retract but synaptic ECM, junctional folds, and acetylcholinesterase (not shown) remained in the empty gutter. This is in contrast to the extending junctional branch $(B)$, where neither junctional folds nor acetylcholinesterase are observed at the extended synaptic ECM region. $G$, Synaptic ECM, junctional folds, and acetylcholinesterase disappear eventually from the empty gutter. These processes
occur after the retraction of an NT branch. 
among synaptic ECM, Schwann cell processes, and NT with repeated in vivo observations in combination with electron microscopy. In addition to finding a specific marker for the terminal Schwann cell membrane, identifying novel ECM molecules preferentially associated with terminal Schwann cells also will be important for further understanding the molecular mechanism of synaptic remodeling. A study toward this aim now is in progress (Astrow et al., 1995).

\section{REFERENCES}

Anzil AP, Bieser A, Wernig A (1984) Light and electron microscopic identification of nerve terminal sprouting and retraction in normal adult frog muscle. J Physiol (Lond) 350:393 399.

Astrow SH, Son YJ, Thompson WJ (1994) Differential neural regulation of a neuromuscular junction-associated antigen in muscle fibers and Schwann cells. J Neurobiol 25:937-952.

Astrow SH, Nguyen MTT, Ko CP (1995) An extracellular matrix glycoprotein associated with neuromuscular junctions and peripheral nerves. Soc Neurosci Abstr 21:1313.

Bailey CH, Kandel ER (1993) Structural changes accompanying memory storage. Annu Rev Physiol 55:397-426.

Balice-Gordon RJ, Lichtman JW (1990) In vivo visualization of the growth of pre- and postsynaptic elements of neuromuscular junctions in the mouse. I Neurosci 10:894-908.

Bunge RP (1994) The role of the Schwann cell in trophic support and regeneration. J Neurol 242[Suppl 1]:S19-S21.

Chen L, Ko CP (1994) Extension of synaptic extracellular matrix during nerve terminal sprouting in living frog neuromuscular junctions. J Neurosci 14:796-808.

Chen L, Folsom DB, Ko CP (1991) The remodeling of synaptic extracellular matrix and its dynamic relationship with nerve terminals at living frog neuromuscular junctions. J Neurosci 11:2920-2930.

Chiu AY, Espinosadelos-Monteros A, Cole RA, Loera S, deVellis J (1991) Laminin and s-laminin are produced and released by astrocytes, Schwann cells and Schwannomas in culture. Glia 4:11-24.

Connor EA, McMahan UJ (1987) Cell accumulation in the junctional region of denervated muscle. J Cell Biol 104:109-120.

Diaz J, Pecot-Dechavassine M (1990) Nerve sprouting induced by a piece of peripheral nerve placed over a normally innervated frog muscle. J Physiol (Lond) 421:123-133.

Engcl AG (1994) The neuromuscular junction. In: Myology, 2nd Ed (Engel AG, Franzini-Armstrong C, eds), pp 261-302. New York: McGraw-Hill.

Gatchalian CL, Schachner M, Sanes JR (1989) Fibroblasts that proliferate near denervated synaptic sites in skeletal muscle synthesize the adhesive molecules tenascin (J1), N-CAM, fibronectin, and a heparin sulfate proteoglycan. J Cell Biol 108:1837-1890.

Herrera AA, Banner LR (1990) The use and effects of vital fluorescent dyes: observation of motor nerve terminals and satellite cells in living frog muscles. J Neurocytol 19:67-83.

Herrera AA, Banner LR, Nagaya N (1990) Repeated, in vivo observation of frog neuromuscular junctions: remodeling involves concurrent growth and retraction. J Neurocytol 9:85-99.

Ko CP (1987) A lectin, peanut agglutinin, as a probe for the extracellular matrix in living neuromuscular junctions. J Ncurocytol 16:567-576.

Ko CP (1991) Peanut agglutinin as a probe for studying remodeling and differentiation of synaptic extracellular matrix at the frog neuromuscu- lar junctions. In: Plasticity of motorneuronal connections (Wernig $\Lambda$, ed), pp 51-62. Amsterdam: Elsevier.

Ko CP, Chen L, Thompson A (1992) Synaptic remodeling revealed by combined video-enhanced fluorescent microscopy and electron microscopy of identified frog neuromuscular junctions. Soc Neurosci Abstr $18: 218$.

Langenfeld-Oster B, Dorlochter M, Wernig A (1993) Regular and photodamage-enhanced remodelling in vitally stained frog and mouse neuromuscular junctions. J Neurocytol 22:517-530.

Lichtman JW, Magrassi L, Purves D (1987) Visualization of neuromuscular junction over periods of several months in living mice. J Neurosci 7:1215-1222.

Magrassi L, Purves D, Lichtman JW (1987) Fluorescent probes that stain living nerve terminals. J Neurosci 7:1207-1214.

Purves D (1989) Assessing some dynamic properties of the living nervous system. Q J Exp Physiol 74:1089-1105.

Purves D, Lichtman JW (1985) Principles of ncural development. Sunderland, MA: Sinauer.

Reichardt LF, Tomaselli KJ (1991) Extracellular matrix molecules and their receptors: functions in neural development. Annu Rev Neurosci 14:531-570.

Reynolds ML, Woolf CJ (1992) Terminal Schwann cells elaborate extensive processes following denervation of the motor end plate. $J$ Neurocytol 21:50-66.

Robbins N, Polak J (1988) Filopodia, lamellipodia, and retractions at mouse neuromuscular junctions. J Neurocytol 17:545-561.

Sanes JR (1989) Extracellular matrix molecules that influence neural development. Annu Rev Neurosci 12:491-516.

Schachner M (1990) Functional implications of glial cell recognition molecules. Semin Neurosci 2:497-507.

Somasekhar T, Ko CP (1991) Effects of denervation on the distribution of peanut agglutinin binding molecules in frog muscles. J Neurocytol 20:65-77.

Son YJ, Thompson WJ (1995a) Schwann cell processes guide regeneration of peripheral axons. Neuron 14:125-132.

Son YJ, Thompson WJ (1995b) Nerve sprouting in muscle is induced and guided by processes extended by Schwann cells. Neuron 14:133-141.

van Mier P, Lichtman JW (1994) Regenerating muscle fibers induce directional sprouting from nearby nerve terminals: studies in living mice. J Neurosci 14:5672-5686.

Wernig A, Herrera AA (1986) Sprouting and remodeling at the nervemuscle junction. Prog Neurobiol 27:251-291.

Wernig A, Pecot-Dechavassine M, Stover H (1980) Sprouting and regression of the nerve at the frog neuromuscular junction in normal conditions and after prolonged paralysis with curare. J Neurocytol 9:277-303.

Wernig A, Anzil AP, Bieser A (1981a) Light and electron microscopic identification of a nerve sprout in muscle of normal adult frog. Neurosci Lett 21:261-266.

Wernig A, Anzil AP, Schwarz U (1981b) Abandoned synaptic sites in muscles of normal adult frog. Neurosci Lett 23:105-110.

Wigston DJ (1989) Remodeling of neuromuscular junction in adult mouse soles. J Neurosci 9:639-647.

Wigston DJ (1990) Repeated in vivo visualization of neuromuscular junctions in adult mouse lateral gastrocnemius. J Neurosci 10:17531761. 